\title{
Laser Spectroscopy of Neutron-Rich Tin Isotopes: A Discontinuity in Charge Radii across the $N=82$ Shell Closure
}

\author{
C. Gorges ${ }^{1, *}$ L. V. Rodríguez,,${ }^{2, \dagger}$ D. L. Balabanski, ${ }^{3}$ M. L. Bissell, ${ }^{4}$ K. Blaum, ${ }^{5}$ B. Cheal, ${ }^{6}$ R. F. Garcia Ruiz,${ }^{7,8,4}$
} G. Georgiev, ${ }^{2}$ W. Gins,${ }^{7,}$ H. Heylen, ${ }^{5,8}$ A. Kanellakopoulos, ${ }^{7}$ S. Kaufmann, ${ }^{1}$ M. Kowalska, ${ }^{8,8}$ V. Lagaki, ${ }^{8,9}$ S. Lechner, ${ }^{8,10}$ B. Maaß, ${ }^{1}$ S. Malbrunot-Ettenauer, ${ }^{8}$ W. Nazarewicz, ${ }^{11}$ R. Neugart, ${ }^{12,5}$ G. Neyens, ${ }^{7}$ W. Nörtershäuser, ${ }^{1, \|}$ P.-G. Reinhard, ${ }^{13}$ S. Sailer, ${ }^{14}$ R. Sánchez,,${ }^{15}$ S. Schmidt, ${ }^{1}$ L. Wehner, ${ }^{12}$ C. Wraith, ${ }^{6}$ L. Xie, ${ }^{4}$ Z. Y. Xu, ${ }^{7}$ X. F. Yang,,${ }^{7,16}$ and D. T. Yordanov ${ }^{2,9}$ ${ }^{1}$ Institut für Kernphysik, Technische Universität Darmstadt, D-64289 Darmstadt, Germany

${ }^{2}$ Institut de Physique Nucléaire, CNRS-IN2P3, Université Paris-Sud, Université Paris-Saclay, 91406 Orsay, France

${ }^{3}$ ELI-NP, Horia Hulubei National Institute for R\&D in Physics and Nuclear Engineering, 077125 Magurele, Romania

${ }^{4}$ School of Physics and Astronomy, The University of Manchester, Manchester M13 9PL, United Kingdom

${ }^{5}$ Max-Planck-Institut für Kernphysik, D-69117 Heidelberg, Germany

${ }^{6}$ Oliver Lodge Laboratory, Oxford Street, University of Liverpool, Liverpool L69 7ZE, United Kingdom

${ }^{7}$ Instituut voor Kern- en Stralingsfysica, KU Leuven, B-3001 Leuven, Belgium

${ }^{8}$ Experimental Physics Department, CERN, CH-1211 Geneva 23, Switzerland

${ }^{9}$ Institut für Physik, Universität Greifswald, 17487 Greifswald, Germany

${ }^{10}$ Technische Universität Wien, Karlsplatz 13, 1040 Wien, Austria

${ }^{11}$ Department of Physics and Astronomy and FRIB Laboratory, Michigan State University, East Lansing, Michigan 48824, USA

${ }^{12}$ Institut für Kernchemie, Universität Mainz, D-55128 Mainz, Germany

${ }^{13}$ Institut für Theoretische Physik II, Universität Erlangen-Nürnberg, 91058 Erlangen, Germany

${ }^{14}$ Technische Universität München, D-80333 Munich, Germany

${ }^{15}$ GSI Helmholtzzentrum für Schwerionenforschung GmbH, D-64291 Darmstadt, Germany

${ }^{16}$ School of Physics and State Key Laboratory of Nuclear Physics and Technology, Peking University, Beijing 100871, China

(Received 22 February 2019; revised manuscript received 22 March 2019; published 16 May 2019)

\begin{abstract}
The change in mean-square nuclear charge radii $\delta\left\langle r^{2}\right\rangle$ along the even- $A$ tin isotopic chain ${ }^{108-134} \mathrm{Sn}$ has been investigated by means of collinear laser spectroscopy at ISOLDE/CERN using the atomic transitions $5 p^{2}{ }^{1} S_{0} \rightarrow 5 p 6 s^{1} P_{1}$ and $5 p^{2}{ }^{3} P_{0} \rightarrow 5 p 6 s{ }^{3} P_{1}$. With the determination of the charge radius of ${ }^{134} \mathrm{Sn}$ and corrected values for some of the neutron-rich isotopes, the evolution of the charge radii across the $N=82$ shell closure is established. A clear kink at the doubly magic ${ }^{132} \mathrm{Sn}$ is revealed, similar to what has been observed at $N=82$ in other isotopic chains with larger proton numbers, and at the $N=126$ shell closure in doubly magic ${ }^{208} \mathrm{~Pb}$. While most standard nuclear density functional calculations struggle with a consistent explanation of these discontinuities, we demonstrate that a recently developed Fayans energy density functional provides a coherent description of the kinks at both doubly magic nuclei, ${ }^{132} \mathrm{Sn}$ and ${ }^{208} \mathrm{~Pb}$, without sacrificing the overall performance. A multiple correlation analysis leads to the conclusion that both kinks are related to pairing and surface effects.
\end{abstract}

DOI: 10.1103/PhysRevLett.122.192502

Introduction.-Nuclear charge radii along isotopic chains are known to exhibit a distinct discontinuity at shell closures [1-3]: Their differential increase per isotope is considerably different before and after the magic number, producing a kink at the shell closure for which a number of theoretical explanations have been proposed. To put it briefly, in a shell-model picture, the kink can be explained by the filling of a new valence neutron shell that has a large

Published by the American Physical Society under the terms of the Creative Commons Attribution 4.0 International license. Further distribution of this work must maintain attribution to the author(s) and the published article's title, journal citation, and DOI. radial extent such that the attractive neutron-proton interaction pulls the protons further out to reduce the symmetry energy. A reduction of the binding energy of the valence neutron also leads to a larger neutron orbital radius and, hence, to a spatially increasing proton distribution. This effect can be related to the spin-orbit force [4-8], which naturally appears in the relativistic mean-field model and often explains the kink in the $\mathrm{Pb}$ chain [9-13]. Other mechanisms impacting the kink in radii involve nuclear compressibility [14], as well as ground-state correlations [15-17] and pairing [18-22] — both increasing the occupation of higher-lying neutron and proton single-particle orbits.

Most of the discussion pertaining to the kink in charge radii was focused on the $N=126$ and $N=28$ shell 
closures in the lead [17,23] and calcium [24,25] region, respectively. For the $N=82$ shell closure, most nonrelativistic nuclear density functional theory (DFT) calculations with Skyrme functionals do not produce a kink along the chain of the tin isotopes, whereas the Fayans functional [20,22], with its extended pairing term, and the M3Y-P6a model [8], with its density-dependent spin-orbit and pairing terms, do. Experimentally, the element with the lowest $Z$ for which nuclear charge radii across the $N=82$ shell closure are available is tellurium $(Z=52)$ [16]. However, here the difference of the slope before and after the kink appears to be significantly decreased compared to the heavier element xenon $(Z=54)$ [26,27]. The question then arises whether this apparent reduction of the $N=82$ kink with a decreasing proton number persists in $Z=50 \mathrm{Sn}$ isotopes, producing no kink like the DFT calculations suggest, or will a rather strong kink appear as suggested by Fayans functional predictions for tin $[20,22]$ and the neighboring Cd chain [28].

The neutron-rich ${ }^{132} \mathrm{Sn}$ is a prototypical doubly magic nucleus, which exhibits many structural similarities to ${ }^{208} \mathrm{~Pb}$ [29]. A strong $N=82$ shell gap in the tin isotopes compared with the neighboring elements [30] has already been established in both mass $[31,32]$ and particle-transfer [29] measurements beyond the magic neutron number $N=82$. The latest data from HIE-ISOLDE [33] and RIKEN [34] gave additional insight into the structure of ${ }^{132} \mathrm{Sn}$, supporting further its doubly magic character. Laser spectroscopic and muonic measurements of nuclear charge radii have previously been performed from ${ }^{108} \mathrm{Sn}$ to ${ }^{132} \mathrm{Sn}$ [35-39], however, partially with lower resolution [39].

Recently, a new quantified energy density functional of the Fayans type has been developed [40] by including information about differential charge radii in the parameteroptimization process, especially those of the heavier calcium isotopes determined in Ref. [25]. The resulting model was found not only to reproduce the charge radii in the calcium isotopes but also to provide an excellent prediction for the iron radii at the $N=28$ shell closure [41], the very light calcium isotopes down to ${ }^{36} \mathrm{Ca}$ [42] and, remarkably, also for the much heavier $\mathrm{Cd}$ isotopes from ${ }^{100} \mathrm{Cd}$ to ${ }^{130} \mathrm{Cd}$ [28].

Motivated by the recent experimental and theoretical developments, we present here new data on charge radii for the even-even ${ }^{108-134} \mathrm{Sn}$ isotopes based on high-precision laser spectroscopic measurements, crossing for the first time the doubly magic $N=82$ shell closure in the tin isotopic chain. The results are discussed in terms of the Fayans and Skyrme models augmented by a statistical multiple correlation analysis for ${ }^{132} \mathrm{Sn}$ and ${ }^{208} \mathrm{~Pb}$ to help understanding the microscopic origin of the kink in charge radii at magic neutron numbers.

Experiment.-Collinear laser spectroscopy was performed on neutron-rich tin isotopes using the atomic transitions $5 p^{2}{ }^{1} S_{0} \rightarrow 5 p 6 s{ }^{1} P_{1}$ ("SP," $452.5 \mathrm{~nm}$ ) and
$5 p^{2}{ }^{3} P_{0} \rightarrow 5 p 6 s^{3} P_{1}$ ("PP," $286.3 \mathrm{~nm}$ ) at the COLLAPS experiment situated at ISOLDE/CERN. Details of the setup are described elsewhere $[3,43]$. In brief, protons from the proton synchrotron booster with an energy of $1.4 \mathrm{GeV}$ and beam currents of up to $2 \mu \mathrm{A}$ hit a neutron converter [44] in close proximity to a uranium carbide $\left(\mathrm{UC}_{x}\right)$ target. Neutron-induced fission provides an efficient production of tin isotopes that were subsequently ionized using resonant laser ionization [45] while at the same time reducing the number of unwanted contaminants. The tin ions were accelerated to an energy of either 40 (SP) or $50 \mathrm{keV}$ (PP), mass separated, and transported to the radio frequency quadrupole cooler and buncher ISCOOL [46], where they were accumulated, cooled, and ejected towards the COLLAPS beam line as ion bunches of typically $5 \mu \mathrm{s}$ pulse length. At COLLAPS, the ion bunches were overlapped with a copropagating single-frequency laser beam. Charge exchange on sodium vapor was performed to populate the lower level of the respective transition. By applying a "Doppler-tuning" potential at the chargeexchange region, the laser frequency in the rest frame of the atoms is varied by the change of velocity. Linearly polarized light was generated by a Sirah Matisse-2 ring laser either in the titanium-sapphire (SP) or in the dye configuration (PP) and frequency doubled in an external cavity (Spectra Physics Wavetrain). The laser was longterm stabilized $(<10 \mathrm{MHz} / h)$ to a wavelength meter (HighFinesse WSU) that has been regularly calibrated using a stabilized helium-neon laser. Reference scans of ${ }^{124} \mathrm{Sn}$ were carried out regularly between the other isotopes. Optical detection of the PP transition was based directly on the fluorescence at $286 \mathrm{~nm}$, while the SP transition was detected by the dominant decay via the $5 p 6 s{ }^{1} P_{1} \rightarrow$ $5 p^{2}{ }^{1} D_{2}$ transition at $326 \mathrm{~nm}$. Figure 1 presents spectra of the neutron-rich even isotopes in the SP transition, with the $x$ axis converted into frequencies relative to the reference isotope ${ }^{124} \mathrm{Sn}$. The signature of the kink at $N=$ 82 is immediately visible, since the shift of the resonance frequency of ${ }^{134} \mathrm{Sn}$ with respect to ${ }^{132} \mathrm{Sn}$ is considerably larger than the very regular differences for $\Delta A=2$ along the isotopes ${ }^{124-132} \mathrm{Sn}$.

Spectra of the even isotopes ${ }^{112-134} \mathrm{Sn}$ were obtained for both transitions, while ${ }^{108,110} \mathrm{Sn}$ were observed only in the PP transition. The laser power was reduced until saturation broadening disappeared. Only the spectrum of ${ }^{134} \mathrm{Sn}$ was recorded with higher power to increase the signal strength, which explains the larger width of the resonance in Fig. 1. In this case, reference spectra of ${ }^{124} \mathrm{Sn}$ were recorded under identical conditions. No systematic shift of ${ }^{124} \mathrm{Sn}$ with respect to its spectra at a reduced laser power was observed. The larger number of spectra taken in the SP transition provided better statistics compared to the PP transition, which explains the larger statistical uncertainties of the PP isotope shifts that are listed in Table I. 


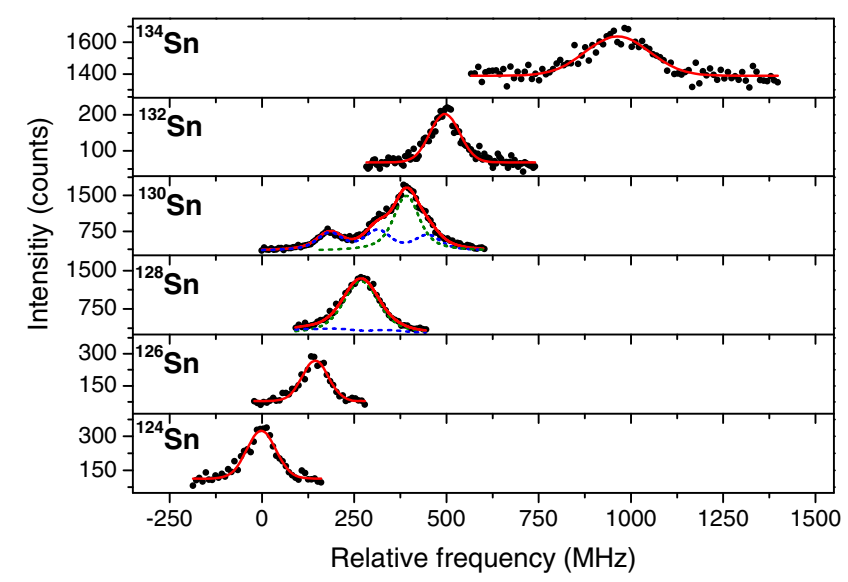

FIG. 1. Optical spectra of even neutron-rich $\mathrm{Sn}$ isotopes for the determination of the isotope shift in the $5 p^{2}{ }^{1} S_{0} \rightarrow 5 p 6 s{ }^{1} P_{1}$ (SP) transition. The number of photomultiplier events is plotted as a function of the Doppler-tuned frequency relative to the resonance center of ${ }^{124} \mathrm{Sn}$. The red line represents the fitted Voigt profile. For ${ }^{128,130} \mathrm{Sn}$, the contribution of an isomer (dashed, blue line) and the $I=0$ ground state (dotted, olive line) are plotted individually, with a significantly smaller isomer-to-ground-state ratio in ${ }^{128} \mathrm{Sn}$.

Experimental results.-The isotope shifts with respect to ${ }^{124} \mathrm{Sn}$ are related to changes in the mean-square charge radii $\delta\left\langle r^{2}\right\rangle^{124, A}$ by

$$
\delta \nu_{i}^{124, A}=\frac{m_{A}-m_{124}}{m_{A} m_{124}} M_{i}+F_{i} \delta\left\langle r^{2}\right\rangle^{124, A},
$$

with the field-shift factor $F$ and the mass shift factor $M$ of the respective transition $i=\mathrm{SP}, \mathrm{PP}$ and the atomic masses $m_{A}$. Spectra in the SP transition were fitted using Voigt profiles with the Lorentzian width fixed to the natural linewidth, with the exception of ${ }^{134} \mathrm{Sn}$ for which it was a free parameter. The FWHM obtained from fitting is below $100 \mathrm{MHz}$ for all isotopes. Resonances in the PP transition had larger width $(\sim 200 \mathrm{MHz})$ and were fitted with a free Lorentzian width. Typical hints of asymmetric profiles due to the charge exchange processes $[3,48]$ were not observed. The spectra of ${ }^{128,130} \mathrm{Sn}$ are afflicted with an underlying partially resolved structure of an isomer. The additional uncertainty caused by insufficient knowledge of the sign of the hyperfine $B$ factor has been estimated by fitting with both possibilities and adding the shift of about $3 \mathrm{MHz}$ to the statistical error. Further details on the fitting routine, especially on combined fitting of the isomers in the two transitions, will be reported in a forthcoming publication.

The largest systematic uncertainty on the isotope shift arises from the relative voltage uncertainties $\Delta U_{\text {acc }} / U_{\text {acc }} \approx$ $\Delta U_{\text {offset }} / U_{\text {offset }} \approx 1.5 \times 10^{-4}$ of the starting potential $U_{\text {acc }}$ at ISCOOL and the Doppler tuning voltage $U_{\text {offset }}$ at the charge exchange cell. Our isotope shifts for ${ }^{112-124} \mathrm{Sn}$ in the SP transition are in excellent agreement, i.e., better than statistically expected, with those reported in Ref. [37].
TABLE I. Isotope shifts $\delta \nu^{124, A}$ and changes in mean-square nuclear charge radii $\delta\left\langle r^{2}\right\rangle^{124, A}$ for even isotopes with mass number $A$ in the $5 p^{2}{ }^{1} S_{0} \rightarrow 5 p 6 s{ }^{1} P_{1}$ (SP) and the $5 p^{2}{ }^{3} P_{0} \rightarrow$ $5 p 6 s^{3} P_{1}$ (PP) transitions. The numbers in parentheses for $\delta \nu^{124, A}$ show statistical and systematic uncertainties. Total uncertainties are listed for $\delta\left\langle r^{2}\right\rangle^{124, A}$, arising primarily from the field-shiftfactor uncertainty, since the voltage-induced systematic uncertainty of the isotope shift largely cancels in the King-plot procedure [47].

\begin{tabular}{lrlrlrl}
\hline \hline$A$ & $\delta \nu_{\mathrm{SP}}^{124, A}$ & $(\mathrm{MHz})$ & \multicolumn{1}{c}{$\delta \nu_{\mathrm{PP}}^{124, A}(\mathrm{MHz})$} & $\delta\left\langle r^{2}\right\rangle^{124, A}$ & $\left(\mathrm{fm}^{2}\right)$ \\
\hline 108 & & & -2416.3 & $(94)(98)$ & -1.081 & $(18)$ \\
110 & & & -2014.9 & $(75)(84)$ & -0.907 & $(11)$ \\
112 & -1383.3 & $(22)(84)$ & -1652.1 & $(56)(71)$ & -0.747 & $(7)$ \\
114 & -1115.3 & $(19)(69)$ & -1335.6 & $(62)(58)$ & -0.605 & $(5)$ \\
116 & -841.8 & $(14)(55)$ & -1007.6 & $(79)(45)$ & -0.461 & $(4)$ \\
118 & -585.6 & $(19)(40)$ & -695.0 & $(67)(33)$ & -0.324 & $(5)$ \\
120 & -360.5 & $(13)(27)$ & -448.6 & $(80)(22)$ & -0.206 & $(5)$ \\
122 & -167.3 & $(16)(13)$ & -206.3 & $(75)(10)$ & -0.097 & $(3)$ \\
126 & 147.2 & $(13)(13)$ & 189.4 & $(47)(12)$ & 0.089 & $(4)$ \\
128 & 273.2 & $(53)(26)$ & 338.9 & $(49)(21)$ & 0.165 & $(10)$ \\
130 & 395.9 & $(65)(37)$ & 486.6 & $(38)(31)$ & 0.240 & $(15)$ \\
132 & 497.7 & $(11)(50)$ & 624.0 & $(32)(42)$ & 0.307 & $(21)$ \\
134 & 981.0 & $(36)(62)$ & 1196.5 & $(74)(51)$ & 0.533 & $(10)$ \\
\hline \hline
\end{tabular}

For the PP transition, we agree statistically sound with values in Ref. [36], but we have a considerable discrepancy on the order of $70 \mathrm{MHz}(\approx 5 \sigma)$ for ${ }^{130,132} \mathrm{Sn}$ with Ref. [39].

The commonly used King-plot analysis (see, e.g., Ref. [49]) was performed for both transitions to determine the atomic factors $M$ and $F$. Therefore, we used muonic data as well as $V$ factors from electron elastic scattering provided in Ref. [27] and our isotope shifts of the stable isotopes. A detailed description of the procedure for the determination of $\delta\left\langle r^{2}\right\rangle$ can be found in Ref. [28], where a similar analysis was performed. Here, we obtained fieldshift factors of $F_{\mathrm{SP}}=2.42(48) \mathrm{GHz} / \mathrm{fm}^{2}$ and $F_{\mathrm{PP}}=$ 2.74(57) $\mathrm{GHz} / \mathrm{fm}^{2}$ for the SP and PP transition, respectively. The corresponding mass-shift factors are $M_{\mathrm{SP}}=$ -494(402) GHzu and $M_{\mathrm{PP}}=-455(479) \mathrm{GHzu}$. The ratio of the field-shift factors is in excellent agreement with the slope of a King plot between the two transitions, in which data from the even isotopes ${ }^{112-134} \mathrm{Sn}$ are used. Moreover, both field-shift factors are in reasonable agreement (within $1 \sigma$ ) with literature values $F_{\mathrm{SP}}=2.24(27) \mathrm{GHz} / \mathrm{fm}^{2}$ [37], $F_{\mathrm{PP}}=2.39(27) \mathrm{GHz} / \mathrm{fm}^{2}$ [37], $F_{\mathrm{PP}}=2.18(17) \mathrm{GHz} / \mathrm{fm}^{2}$ [27], and $F_{\mathrm{PP}}=3.3(3) \mathrm{GHz} / \mathrm{fm}^{2}$ [36], which were either based on a Dirac-Fock calculation in the $\mathrm{Sn}^{+}$ $6 s^{2} S_{1 / 2} \rightarrow 6 p^{2} P_{3 / 2}(\lambda=645.6 \mathrm{~nm})$ transition, projected onto the respective transitions in neutral $\mathrm{Sn}$ with a Kingplot procedure [37], by the analysis of the hyperfine splitting in the $645.6 \mathrm{~nm}$ line in $\mathrm{Sn}^{+}$[36], or by a similar King-plot procedure as performed here [27].

Changes in $\left\langle r^{2}\right\rangle$ were determined from both transitions and are in good agrement with each other as well as with 
values available in Ref. [27]. The weighted averages from the two transitions are listed in Table I. Uncertainties were taken as the minimum from the two transitions but not further reduced. In ${ }^{134} \mathrm{Sn}$, the error has been increased by $20 \%$ to incorporate a small difference in the resulting charge radii.

The evolution of the nuclear charge radii of the even tin isotopes is shown in Fig. 2, based on the reference value of $\left\langle r^{2}\right\rangle_{\mu \mathrm{e}}^{1 / 2}=R_{k \alpha}^{\mu} / V_{2}=4.675(1) \mathrm{fm}$ of ${ }^{124} \mathrm{Sn}$ [27]. Below ${ }^{132} \mathrm{Sn}$, the trend is almost linear, with a small curvature. A clear kink is seen at $N=82$ that is indicative of a neutron shell closure and compared with the kink at ${ }^{208} \mathrm{~Pb}$ shown in the inset.

Theoretical analysis.-To understand the experimental findings, we employed nuclear DFT [50] at a spherical Hartree-Fock-Bogoliubov (HFB) level as in Ref. [42]. Calculations were carried out using two different energy functionals. The parametrization SV-min [51] is based on the widely used Skyrme functional [50], while the functional $\mathrm{Fy}(\Delta r, \mathrm{HFB})$ recently developed in Ref. [42] is the Fayans functional involving gradient terms in surface and pairing energies [20,52]. Both parametrizations, SV-min and $\mathrm{Fy}(\Delta r, \mathrm{HFB})$, were fitted to the same large set of ground-state data achieving the same high quality in reproducing them. As discussed in Refs. [19,20,40], the density-dependent Fayans pairing functional involving the gradient term explains the odd-even staggering effect in charge radii by producing a direct coupling between the proton density and the neutron pair density. The blocking effect $[53,54]$ in an odd- $N$ nucleus yields a reduced neutron pairing density, which in turn impacts the proton density and, hence, the charge radius. This argument does not apply

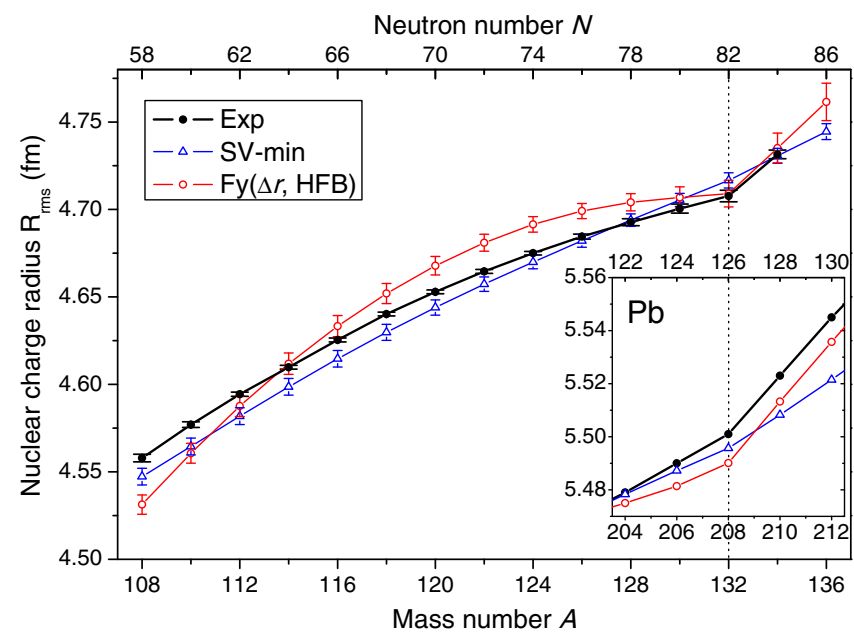

FIG. 2. Evolution of the nuclear charge radii along the tin isotopic chain from the experiment (circles) and from DFT calculations using the SV-min (triangles) and Fy $(\Delta r, \mathrm{HFB})$ (dots) functionals. The experimental and predicted charge radii at the kink at ${ }^{208} \mathrm{~Pb}$ are shown in the inset. Experimental data for ${ }^{204-212} \mathrm{~Pb}$ are taken from Ref. [27]. to open-shell systems, in which deformation effects on odd$N$ nuclei can be dramatic, as is observed, e.g., in the mercury chain [55-57].

To facilitate the discussion, we introduce the threepoint indicator of an observable $\mathcal{O}: \Delta_{k n}^{(3)} \mathcal{O}(Z, N) \equiv$ $\frac{1}{2}[\mathcal{O}(Z, N+k)-2 \mathcal{O}(Z, N)+\mathcal{O}(Z, N-k)]$, where the odd-even staggering in $\mathcal{O}$ corresponds to $k=1$ while the curvature (or kink) parameter is given by $k=2$. In the case of charge radii $(\mathcal{O}=r)$, the staggering $\Delta_{1 n}^{(3)} r(Z, N)$ is usually negative for even- $N$ semimagic systems; i.e., the charge radii of odd- $N$ proton-magic nuclei are usually smaller than the average of their even- $N$ neighbors. Since in the Fayans model the magnitude of $\Delta_{1 n}^{(3)} r$ is explained by the reduced neutron pairing in odd- $N$ nuclei, this suggests a simple explanation of the kink in charge radii at magic numbers. Indeed, for the magic neutron number $N$, the neutron pairing is absent, while it is significant for the $N \pm 2$ neighbors, resulting in a distinct kink $\Delta_{2 n}^{(3)} r(Z, N)>0$.

The results of SV-min and Fy $(\Delta r$, HFB $)$ for the charge radii along the tin isotopic chain are shown in Fig. 2; there is a significant difference between the two models. Namely, $\mathrm{SV}$-min produces a flat trend $\left(\Delta_{2 n}^{(3)} r=0.0014 \mathrm{fm}\right)$, while Fy $(\Delta r, \mathrm{HFB})$ predicts a kink of $0.0119 \mathrm{fm}$ for ${ }^{132} \mathrm{Sn}$ in accordance with the experiment $(0.0078 \mathrm{fm})$ and earlier Fayans-model predictions [20,22]. This is similar for the parabolic behavior of the radii below the $N=82$ shell closure, which is visible in the experimental data, almost absent in SV-min, and overexpressed in Fy $(\Delta r$, HFB $)$.

Figure 2 compares experimental data for the kinks in ${ }^{132} \mathrm{Sn}$ and ${ }^{208} \mathrm{~Pb}$ (inset) with our SV-min and Fy $(\Delta r, \mathrm{HFB})$ predictions. It is seen that also in this case SV-min $\left(\Delta_{2 n}^{(3)} r=0.0021 \mathrm{fm}^{2}\right)$ significantly underestimates the kink (experiment, $\left.0.0043 \mathrm{fm}^{2}\right)$ while $\mathrm{Fy}(\Delta r, \mathrm{HFB})$ slightly overestimates it $\left(0.0075 \mathrm{fm}^{2}\right)$. This result is consistent with our working hypothesis about the nature of the kink and the systematic analysis of Ref. [40]: $\Delta_{1 n}^{(3)} r(Z, N)$ is primarily driven by the gradient term in the Fayans pairing functional.

As mentioned above, several mechanisms responsible for the kink in charge radii have been proposed. A comprehensive analysis requires the control of all ingredients to a nuclear model simultaneously, which can be achieved in a systematic fashion by statistical linear leastsquare regression analysis [58]. Specifically, we look at the statistical correlations between the kinks in radii and model parameters as well as between the kinks and selected observables. However, in view of 13-14 model parameters, it is more efficient to look at multiple correlation coefficients (MCCs) of a kink with groups of model parameters [59]. Here, we closely follow the methodology laid out in Ref. [60]. The MCCs range from 0 to 1 , where 0 implies that the kink is uncorrelated with the group of parameters or observables and the value of 1 means total correlation. 
The statistical analysis has been performed for four groups of parameters: $P$ (pairing, all parameters defining the pairing functional); $S$ (surface, all parameters pertaining to terms with gradients of density); LS (spin-orbit, isoscalar, and isovector spin-orbit coupling constants); and SE (symmetry energy $J$, slope of symmetry energy $L$ ). These groups are chosen because they either show large MCC or had been previously discussed in the context of the kinks in radii. We do not discuss volume parameters, which have been found to have a rather small impact on the kinks. We recall that both parametrizations SV-min and $\mathrm{Fy}(\Delta r, \mathrm{HFB})$ are fitted to basically the same dataset; hence, differences between their results are primarily related to the form of underlying functionals. Details on the two energy functionals are provided in Supplemental Material [61].

Figure 3 displays the results of our statistical analysis. The first four panels show the MCCs of the radius kinks in ${ }^{132} \mathrm{Sn}$ and ${ }^{208} \mathrm{~Pb}$ with the groups of model parameters. For both $\mathrm{SV}$-min and $\mathrm{Fy}(\Delta r, \mathrm{HFB})$, pairing and surface groups are the primary drivers. The common $\mathrm{MCC}(P+S)$ is smaller than the sum of the separate MCCs, which indicates the expected strong intercorrelations between pairing and surface parameters. Spin-orbit and symmetry-energy groups are less important, especially for $\Delta_{2 n}^{(3)} r\left({ }^{208} \mathrm{~Pb}\right)$ in $\mathrm{Fy}(\Delta r, \mathrm{HFB})$. Altogether, the MCCs with model parameters quantitatively confirm our scenario that the pairing functional plays a leading role in producing the kinks.

The last two panels in Fig. 3 show the MCCs of the kinks with the groups of odd-even staggering observables $\Delta_{1}^{(3)} E$ and $\Delta_{1}^{(3)} r$ calculated for neutrons and protons. It becomes clear that the kinks in charge radii in magic nuclei are statistically correlated with odd-even staggering of radii

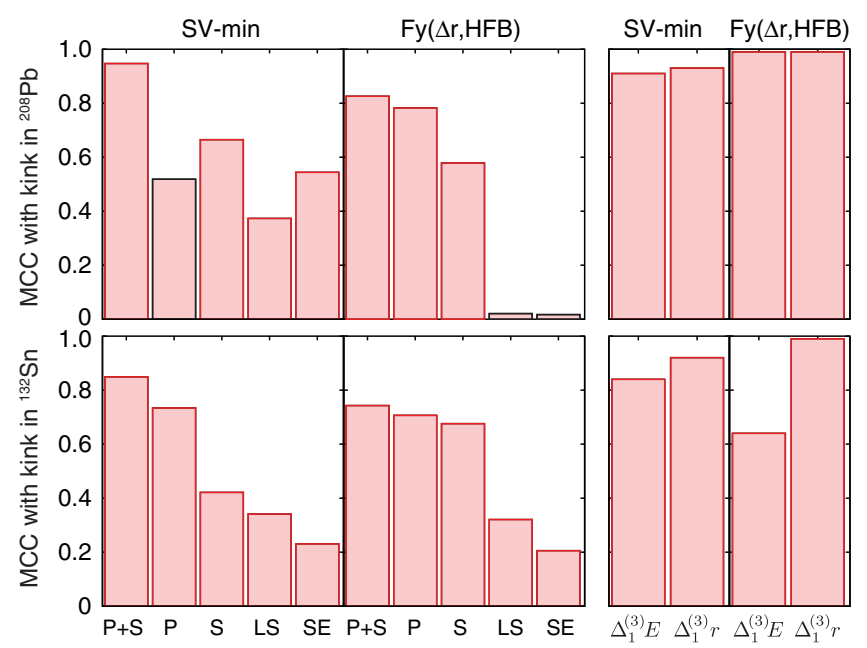

FIG. 3. Multiple correlation coefficient of the radius kinks $\Delta_{2 n}^{(3)} r$ in ${ }^{132} \mathrm{Sn}$ and ${ }^{208} \mathrm{~Pb}$ with groups of model parameters (left) and groups of odd-even staggerings in binding energy, $\Delta_{1}^{(3)} E$, and charge radii, $\Delta_{1}^{(3)} r$, (right) calculated with $\mathrm{SV}$-min and Fy $(\Delta r, \mathrm{HFB})$. For details, see Supplemental Material [61]. and odd-even staggering of binding energies, both significantly impacted by nucleonic pairing. It should be emphasized that $\mathrm{Fy}(\Delta r, \mathrm{HFB})$ provides binding energies $\left(S_{2 n}\right.$ and $\left.S_{n}\right)$ along the Sn chain comparably well as SV-min.

Conclusions.-The changes in the mean-square nuclear charge radius of the even tin isotopic chain ${ }^{108-134} \mathrm{Sn}$ have been determined by means of collinear laser spectroscopy. With the first charge-radius measurement of a neutronrich $\mathrm{Sn}$ isotope beyond $N=82$ and corrected values for ${ }^{130,132} \mathrm{Sn}$, we revealed a characteristic kink at ${ }^{132} \mathrm{Sn}$ of similar strength as in tellurium. Nuclear density functional theory calculations using the Fayans functional $\mathrm{Fy}(\Delta r$, HFB $)$ produce this kink, as well as the kink at ${ }^{208} \mathrm{~Pb}$, in reasonable agreement with data without the additional tuning of parameters, contrary to the conventional Skyrme functional SVmin, which grossly underestimates the kink's magnitude.

A multiple-correlation analysis shows that the common mechanism primarily responsible for producing odd-even staggering of charge radii in semimagic isotopic chains and the kinks at magic numbers is the reduction of pairing in odd$N$ and magic nuclei. The magnitude of this effect is vastly different for SV-min and $\mathrm{Fy}(\Delta r, \mathrm{HFB})$. In this respect, the gradient coupling term in the Fayans functional is essential to provide agreement with the experiment [19,20,22,40,42]. It is quite remarkable that the $\mathrm{Fy}(\Delta r, \mathrm{HFB})$ has now been successful in describing radii trends from calcium up into the $\mathrm{Sn}$ and even $\mathrm{Pb}$ regions. Clearly, new data on charge radii in exotic nuclei will continue to be extremely valuable for developing better pairing functionals.

We thank the ISOLDE technical group for their professional assistance. This work has been supported by the Max-Planck Society, the German Federal Ministry for Education and Research under Contract No. 05P15RDCIA; the Helmholtz International Center for FAIR (HIC for FAIR) within the LOEWE program by the State of Hesse; the Belgian IAP Project No. P7/12, the FWO-Vlaanderen; GOA 15/010 from KU Leuven; the European Union seventh framework through ENSAR under Contract No. 262010; the Science and Technology Facilities Council (ST/L005670/ 1 and ST/L005794/1), and by the Office of Science, U.S. Department of Energy under Grants No. DE-SC0013365 and No. DE-SC0018083 (NUCLEI SciDAC-4 Collaboration). M. K. acknowledges funding from ERC Starting Grant No. 640465 and D. L. B. from the EU Development Fund and Competitiveness Operational Program for the ELI-NP Project Phase II (1/07.07.2016, COP, ID1334).

C. G. and L. V. R. contributed equally to this work.

*Present address: Institut für Kernchemie, Universität Mainz, D-55128 Mainz, Germany.

${ }^{\dagger}$ Max-Planck-Institut für Kernphysik, D-69117 Heidelberg, Germany. 
${ }^{\ddagger}$ Department of Physics, University of Jyväskylä, PB 35 (YFL) FIN-40351 Jyväskylä, Finland.

${ }^{\S}$ DNPC, Université de Geneve, CH-1205 Geneva, Switzerland.

"wnoertershaeuser@ikp.tu-darmstadt.de

yordanov@ipno.in2p3.fr

[1] P. Campbell, I. Moore, and M. Pearson, Prog. Part. Nucl. Phys. 86, 127 (2016).

[2] C. Thibault, F. Touchard, S. Büttgenbach, R. Klapisch, M. de Saint Simon, H. T. Duong, P. Jacquinot, P. Juncar, S. Liberman, P. Pillet, J. Pinard, J. L. Vialle, A. Pesnelle, and G. Huber, Phys. Rev. C 23, 2720 (1981).

[3] A. Mueller, F. Buchinger, W. Klempt, E. Otten, R. Neugart, C. Ekström, and J. Heinemeier, Nucl. Phys. A403, 234 (1983).

[4] P.-G. Reinhard and H. Flocard, Nucl. Phys. A584, 467 (1995).

[5] J. Erler, P. Klüpfel, and P.-G. Reinhard, J. Phys. G 38, 033101 (2011).

[6] P. M. Goddard, P. D. Stevenson, and A. Rios, Phys. Rev. Lett. 110, 032503 (2013).

[7] H. Nakada and T. Inakura, Phys. Rev. C 91, 021302(R) (2015).

[8] H. Nakada, Phys. Rev. C 92, 044307 (2015).

[9] M. M. Sharma, G. A. Lalazissis, and P. Ring, Phys. Lett. B 317, 9 (1993).

[10] M. M. Sharma, G. Lalazissis, J. König, and P. Ring, Phys. Rev. Lett. 74, 3744 (1995).

[11] S. E. Agbemava, A. V. Afanasjev, D. Ray, and P. Ring, Phys. Rev. C 89, 054320 (2014).

[12] A. V. Afanasjev and S. E. Agbemava, Phys. Rev. C 93, 054310 (2016).

[13] J.-P. Ebran, A. Mutschler, E. Khan, and D. Vretenar, Phys. Rev. C 94, 024304 (2016).

[14] R. Niembro, S. Marcos, M. López-Quelle, and L. N. Savushkin, Phys. At. Nucl. 75, 269 (2012).

[15] M. Bender, G. F. Bertsch, and P.-H. Heenen, Phys. Rev. C 73, 034322 (2006).

[16] J. Libert, B. Roussiére, and J. Sauvage, Nucl. Phys. A786, 47 (2007)

[17] T. E. Cocolios et al., Phys. Rev. Lett. 106, 052503 (2011).

[18] N. Tajima, P. Bonche, H. Flocard, P.-H. Heenen, and M. S. Weiss, Nucl. Phys. A551, 434 (1993).

[19] S. Fayans and D. Zawischa, Phys. Lett. B 383, 19 (1996).

[20] S. Fayans, S. Tolokonnikov, E. Trykov, and D. Zawischa, Nucl. Phys. A676, 49 (2000).

[21] S. V. Tolokonnikov and E. E. Saperstein, Phys. At. Nucl. 73, 1684 (2010).

[22] E. E. Saperstein and S. V. Tolokonnikov, Phys. At. Nucl. 74, 1277 (2011).

[23] M. Anselment, W. Faubel, S. Göring, A. Hanser, G. Meisel, H. Rebel, and G. Schatz, Nucl. Phys. A451, 471 (1986).

[24] L. Vermeeren, R. E. Silverans, P. Lievens, A. Klein, R. Neugart, C. Schulz, and F. Buchinger, Phys. Rev. Lett. 68, 1679 (1992).

[25] R. F. Garcia Ruiz et al., Nat. Phys. 12, 594 (2016).

[26] W. Borchers, E. Arnold, W. Neu, R. Neugart, K. Wendt, and G. Ulm, Phys. Lett. B 216, 7 (1989).

[27] G. Fricke and K. Heilig, Nuclear Charge Radii, Group I: Elementary Particles, Nuclei and Atoms Vol. 20 (Springer, New York, 2004).
[28] M. Hammen et al., Phys. Rev. Lett. 121, 102501 (2018).

[29] K. L. Jones et al., Nature (London) 465, 454 (2010).

[30] D. Atanasov et al., Phys. Rev. Lett. 115, 232501 (2015).

[31] M. Dworschak, G. Audi, K. Blaum, P. Delahaye, S. George, U. Hager, F. Herfurth, A. Herlert, A. Kellerbauer, H.-J. Kluge, D. Lunney, L. Schweikhard, and C. Yazidjian, Phys. Rev. Lett. 100, 072501 (2008).

[32] J. Hakala, J. Dobaczewski, D. Gorelov, T. Eronen, A. Jokinen, A. Kankainen, V. S. Kolhinen, M. Kortelainen, I. D. Moore, H. Penttilä, S. Rinta-Antila, J. Rissanen, A. Saastamoinen, V. Sonnenschein, and J. Äystö, Phys. Rev. Lett. 109, 032501 (2012).

[33] D. Rosiak et al. (MINIBALL and HIE-ISOLDE Collaborations), Phys. Rev. Lett. 121, 252501 (2018).

[34] J. Yasuda et al., Phys. Rev. Lett. 121, 132501 (2018).

[35] P. E. G. Baird, S. A. Blundell, G. Burrows, C. J. Foot, G. Meisel, D. N. Stacey, and G. K. Woodgate, J. Phys. B 16, 2485 (1983).

[36] M. Anselment, K. Bekk, A. Hanser, H. Hoeffgen, G. Meisel, S. Goring, H. Rebel, and G. Schatz, Phys. Rev. C 34, 1052 (1986).

[37] J. Eberz, U. Dinger, G. Huber, H. Lochmann, R. Menges, G. Ulm, R. Kirchner, O. Klepper, T. U. Kühl, and D. Marx, Z. Phys. A 326, 121 (1987).

[38] C. Piller, C. Gugler, R. Jacot-Guillarmod, L. A. Schaller, L. Schellenberg, H. Schneuwly, G. Fricke, T. Hennemann, and J. Herberz, Phys. Rev. C 42, 182 (1990).

[39] F. L. Blanc, L. Cabaret, E. Cottereau, J. E. Crawford, S. Essabaa, J. Genevey, R. Horn, G. Huber, J. Lassen, J. K. P. Lee, G. L. Scornet, J. Lettry, J. Obert, J. Oms, A. Ouchrif, J. Pinard, H. Ravn, B. Roussière, J. Sauvage, and D. Verney, Phys. Rev. C 72, 034305 (2005).

[40] P.-G. Reinhard and W. Nazarewicz, Phys. Rev. C 95, 064328 (2017).

[41] K. Minamisono, D. M. Rossi, R. Beerwerth, S. Fritzsche, D. Garand, A. Klose, Y. Liu, B. Maßß, P. F. Mantica, A. J. Miller, P. Müller, W. Nazarewicz, W. Nörtershäuser, E. Olsen, M. R. Pearson, P.-G. Reinhard, E. E. Saperstein, C. Sumithrarachchi, and S. V. Tolokonnikov, Phys. Rev. Lett. 117, 252501 (2016).

[42] A. J. Miller, K. Minamisono, A. Klose, D. Garand, C. Kujawa, J. D. Lantis, Y. Liu, B. Maaß, P. F. Mantica, W. Nazarewicz, W. Nörtershäuser, S. V. Pineda, P.-G. Reinhard, D. M. Rossi, F. Sommer, C. Sumithrarachchi, A. Teigelhöfer, and J. Watkins, Nat. Phys. 15, 432 (2019).

[43] R. Neugart, J. Billowes, M. L. Bissell, K. Blaum, B. Cheal, K. T. Flanagan, G. Neyens, W. Nörtershäuser, and D. T. Yordanov, J. Phys. G 44, 064002 (2017).

[44] R. Catherall, J. Lettry, S. Gilardoni, and U. Köster, Nucl. Instrum. Methods Phys. Res., Sect. B 204, 235 (2003).

[45] B. A. Marsh, Rev. Sci. Instrum. 85, $02 B 923$ (2014).

[46] H. Franberg, P. Delahaye, J. Billowes, K. Blaum, R. Catherall, F. Duval, O. Gianfrancesco, T. Giles, A. Jokinen, M. Lindroos, D. Lunney, E. Mane, and I. Podadera, Nucl. Instrum. Methods Phys. Res., Sect. B 266, 4502 (2008).

[47] A. Klein, B. Brown, U. Georg, M. Keim, P. Lievens, R. Neugart, M. Neuroth, R. Silverans, and L. Vermeeren (ISOLDE Collaboration), Nucl. Phys. A607, 1 (1996).

[48] N. Bendali, H. T. Duong, P. Juncar, J. M. S. Jalm, and J. L. Vialle, J. Phys. B 19, 233 (1986). 
[49] G. Fricke, C. Bernhardt, K. Heilig, L. Schaller, L. Schellenberg, E. Shera, and C. Dejager, At. Data Nucl. Data Tables 60, 177 (1995).

[50] M. Bender, P.-H. Heenen, and P.-G. Reinhard, Rev. Mod. Phys. 75, 121 (2003).

[51] P. Klüpfel, P.-G. Reinhard, T. J. Bürvenich, and J. A. Maruhn, Phys. Rev. C 79, 034310 (2009).

[52] S. A. Fayans, JETP Lett. 68, 169 (1998).

[53] P. Ring and P. Schuck, The Nuclear Many-Body Problem (Springer, Heidelberg, 1980).

[54] T. Duguet, P. Bonche, P.-H. Heenen, and J. Meyer, Phys. Rev. C 65, 014310 (2001).

[55] J. Bonn, G. Huber, H.-J. Kluge, L. Kugler, and E. Otten, Phys. Lett. 38B, 308 (1972).
[56] T. Kühl, P. Dabkiewicz, C. Duke, H. Fischer, H. J. Kluge, H. Kremmling, and E. W. Otten, Phys. Rev. Lett. 39, 180 (1977).

[57] B. A. Marsh et al., Nat. Phys. 14, 1163 (2018).

[58] J. Dobaczewski, W. Nazarewicz, and P.-G. Reinhard, J. Phys. G 41, 074001 (2014).

[59] P. D. Allison, Multiple Regression: A Primer (Sage Publications, Thousand Oaks, 1998).

[60] B. Schuetrumpf, W. Nazarewicz, and P.-G. Reinhard, Phys. Rev. C 96, 024306 (2017).

[61] See Supplemental Material at http://link.aps.org/ supplemental/10.1103/PhysRevLett.122.192502 for details on the energy density functionals discussed in the paper and on the groups of parameters used in the statistical analysis. 